\title{
Brain MRI diffusion-weighted imaging in patients with classical phenylketonuria
}

\author{
Renzo Manara - Alessandro P. Burlina • Valentina Citton - Mario Ermani • \\ Francesco Vespignani · Carla Carollo • Alberto B. Burlina
}

Received: 24 January 2009 / Accepted: 13 July 2009 /Published online: 4 August 2009

(C) Springer-Verlag 2009

\begin{abstract}
Introduction The aim of this study was to grade magnetic resonance white matter abnormalities (WMAs) of classical phenylketonuria (cPKU) patients treated from birth and to compare sensitivity and specificity of T2-weighted and diffusion-weighted images (DWI).

Methods Twenty early-treated cPKU patients still on a lowphenylalanine diet (12 males; mean age 21.2 years) and 26 normal subjects (ten males; mean age 25.1 years) were enrolled. Typical T2- and diffusion-weighted WMAs were semiquantitatively graded according to Thompson score (TS). Besides, a regional magnetic resonance imaging (MRI) score (mTS) was developed according to extension and intensity of WMAs. Phenylalanine and tyrosine plasma
\end{abstract}

R. Manara $\cdot$ V. Citton $\cdot$ C. Carollo

Neuroradiologic Unit,

University Hospital of Padua,

Padua, Italy

\section{A. P. Burlina $\cdot$ M. Ermani}

Neurological Clinic, Department of Neuroscience,

University Hospital of Padua,

Padua, Italy

F. Vespignani · A. B. Burlina

Metabolic Diseases Unit, Department of Paediatrics,

University Hospital of Padua,

Padua, Italy

\section{Citton $(\bowtie)$}

Neuroradiologic Unit, University Hospital of Padua,

Via Giustiniani 5,

35128 Padua, Italy

e-mail: valentina.citton@sanita.padova.it

Present Address:

A. P. Burlina

Neurological Unit, San Bassiano Hospital,

Bassano del Grappa, Italy concentrations before performing MRI and the amino acid mean levels collected the year before MRI (Tyr year and Phe $_{\text {year }}$ ) were measured.

Results No patient with $\mathrm{Phe}_{\text {year }}$ concentration below $460 \mu \mathrm{mol} / \mathrm{L}$ showed WMAs. In cPKU patients, TS and mTS were significantly higher on DWI than on T2 images (3.50 vs 2.65 and 23.65 vs 15.85 , respectively, $p<0.002$, Wilcoxon test). All controls were scored 0 on DWI, while in T2 images, TS and mTS were 0.19 and 1.70. DWI evaluated by mTS disclosed a frontotemporal, occipital, and parietal WM progressive involvement. TS and mTS, both on T2 images and on DWI, showed no correlation with tyrosine while they proved to have a strong correlation with phenylalaninemia and an excellent one with $\mathrm{Phe}_{\text {year }}$ levels. Conclusion Among the different MR sequences, DWI seems to be the most sensitive and reliable in detecting and grading the typical WMAs of cPKU patients.

Keywords Phenylketonuria $\cdot$ Metabolic disease ·

Magnetic resonance imaging $\cdot$ Diffusion-weighted imaging

\begin{tabular}{|c|c|}
\hline \multicolumn{2}{|c|}{ Abbreviations } \\
\hline $\mathrm{cPKU}$ & Classical phenylketonuria \\
\hline Phe & Phenylalanine \\
\hline Tyr & Tyrosine \\
\hline TS & Thompson score \\
\hline mTS & Modified Thompson score \\
\hline Phe $_{\text {year }}$ & $\begin{array}{l}\text { Mean phenylalanine plasma levels collected } \\
\text { in the year before MRI }\end{array}$ \\
\hline WMAs & White matter abnormalities \\
\hline PKU & Phenylketonuria \\
\hline MRI & Magnetic resonance imaging \\
\hline FLAIR & Fluid-attenuated inversion recovery \\
\hline $\mathrm{ADC}$ & Apparent diffusion coefficient \\
\hline DWI & Diffusion-weighted imaging \\
\hline
\end{tabular}




\section{Introduction}

Phenylketonuria (PKU) is a severe autosomal recessive disorder due to phenylalanine (Phe) hydroxylase deficiency [1-3]. Early detection of PKU patients through neonatal screening allows an early dietary treatment which prevents most of the neurological abnormalities [3, 4]. Nevertheless, adolescent and adult treated PKU patients show white matter abnormalities (WMAs) characterized by hyperintense parieto-occipital lesions on T2 images or by periventricular signal abnormalities. These can appear either as band-like or patchy and partly confluent in an irregular pattern or they can sometimes seem like little flames extending from the ventricular border [1-5]. These abnormalities may extend to subcortical or frontal WM as well as to the corpus callosum, the fornix, and the corticospinal tract; additional isolated WM spots may occasionally be present [3]. Patients with more severe alterations on T2 imaging also show slightly hypointense areas on T1 imaging. In 1993, Thompson et al. [6] suggested a dedicated scale to evaluate WMAs on T2weighted images in PKU patients and found a significant correlation between white matter involvement and plasmatic Phe concentration.

Recently, a restricted diffusion of water molecules has been reported in the areas of WMAs [1, 3, 7, 8]. Therefore, they appear hyperintense on diffusion-weighted imaging (DWI) in an otherwise rather homogeneously gray brain parenchyma. This signal pattern not only has become a typical magnetic resonance (MR) feature in hyperphenylalaninemic disorders but also has arisen many questions on the nature of WM changes [1, 5, 7, 9, 10]. As a matter of fact, there are no studies on the histopathological findings in early-treated patients, as the dietary treatment was introduced only in the early 1960s and the oldest patients have reached middle age [7]. Therefore, as far as we know, the available brain pathological reports refer to untreated patients $[11,12]$. Several MR techniques have been applied "in vivo" in order to understand the nature of WMAs. Spectroscopy, DWI, multiecho sequences, and diffusion tensor imaging have revealed variations in metabolite concentrations, decrease of apparent diffusion coefficient (ADC) values, changes in $\mathrm{T} 2$ relaxivity, or abnormalities in white matter anisotropy, thus providing interesting clues. The MR findings in the involved WM areas have led to hypothesize the presence of cytotoxic edema due to the reduction of the $\mathrm{Na}^{+} \mathrm{K}^{+}$-ATPase activity [11], dysmyelination associated to intramyelinic edema [1, 9], status spongiosus, and swelling/separation of myelin sheaths with bound water trapped in myelin sheaths and increased amount of "free" water within sheaths and vacuoles [7]. Nevertheless, despite the consistent bulk of available MR data, the ultimate histological changes and the neurochem- ical mechanism of this selective vulnerability has not been yet unraveled.

Several studies have shown that WMAs do not usually appear in young children under a strict dietary control or with milder variants of PKU and that they are considerably more evident in those who have not properly followed the phenylalanine-restricted diet. Follow-up scans after resumption of a strict diet showed that in some patients, the abnormalities had resolved after a few months but in others, they had not $[3,7,13]$. The clinical meaning of WMAs in PKU patients has still to be clarified and even if they do not seem to correlate with the neurological status, long-term follow-up data are still lacking and the rigorousness of dietary control in adult PKU patients is still a matter of discussion. In this context, we require simple and reliable parameters for following up PKU patients and blood Phe samples do not completely full-fit these characteristics since they suffer from high intraindividual variability due to contingent dietary factors. All the aforementioned MR techniques have shown a strict relationship between plasma Phe level and the measured parameters [1-5, 7], thus confirming the previous report of Thompson on T2-weighted images, but most of these MR studies require a sophisticated software, are time consuming, or do not represent the whole brain since applied on single voxels. Therefore, a semiquantitative score maintains, in a clinical setting, several advantages as it is less time consuming, and it may be applied on plain films even years after the MR examination. On the other hand, although many papers have outlined the peculiar signal pattern of T2 and DWI within the involved WM, no authors have tried a comparison of these techniques in order to understand which is more sensitive or specific while assessing PKU-related WMAs.

The aim of this study is to verify the accuracy of DWI in comparison to $\mathrm{T} 2$ imaging in evaluating WMAs by means of the semiquantitative score proposed by Thompson [6] applied on a highly homogeneous PKU cohort. Moreover, we suggested a regional MR score in order to closely follow-up the characteristic WMAs of patients affected by the classical form of PKU (cPKU).

\section{Materials and methods}

\section{Subjects}

Between November 2006 and June 2007, 21 patients with classical PKU (nine females and 12 males; mean age 21.2 years; age range 10-30 years), followed at our Division of Inherited Metabolic Disorders and Neurological Clinic, were recruited to take part in the study. All patients were identified by neonatal screening and put on low phenylalanine diet with a phenylalanine-free amino acid 
mixture, vitamins, minerals, and trace elements since birth. None had a history or clinical evidence of neurological deterioration. Even if there is no international agreement about Phe concentrations that may be regarded as "harmless" to the brain, we recommended Phe below $480 \mu \mathrm{mol} / \mathrm{L}$ for patients between 6 and 12 years and below $700 \mu \mathrm{mol} / \mathrm{L}$ for adolescents and adults. However, maintenance of good biochemical control in this group of patients is difficult and may result in significant difference between samples. Plasma and blood spot Phe concentrations were analyzed by tandem mass spectrometry (MS/MS). The frequency of Phe measurements varied from every month $(<14$ years) to every 2 months ( $>14$ years). Both plasma phenylalanine levels (Phe) and mean Phe concentrations during the year before MR imaging $\left(\mathrm{Phe}_{\text {year }}\right.$ ) have been measured in all cPKU patients, except one, and are shown in Table 1.

One patient presented severe artifacts on DWI, due to braces, which did not allow a correct evaluation of signal abnormalities and was therefore excluded from the present study. Twenty patients were included in the statistical analysis. Significant demographic and biochemical data (phenylalanine and tyrosine (Tyr) blood levels) concerning our PKU sample are shown in Table 1.

Twenty-six age-matched subjects (ten males and 16 females; mean age 25.1; age range 16-31 years) were enrolled for MR reference data. These subjects underwent brain MRI for the following reasons: 23 because of tensiontype headache, two suffered from migraine, and one presented Chiari malformation type 1 ; patients with a history of malignancy or previous brain disease were excluded.

Informed consent was obtained from patients and parents of minors.

\section{Brain MRI measurements}

The study was performed with a 1.0-T MR scanner (Marconi Picker, Polaris) with a standard quadrature transmit/receive birdcage head coil. Imaging protocol was as follows:

1. Sagittal spin echo (SE) T1-weighted sequence [field of view (FOV) $24 \mathrm{~cm}$, matrix $192 \times 256$, thickness $6 \mathrm{~mm}$, gap $0.5 \mathrm{~mm}, 12 \mathrm{~ms} / 434 \mathrm{~ms} / 2$ (TE/TR/Nex); acquisition time $3 \mathrm{~min} 28 \mathrm{~s}$ ]

2. Axial SE T1-weighted magnetization-prepared spinecho sequence [FOV $25 \mathrm{~cm}$, matrix $192 \times 256,16 \mathrm{~ms} /$ $723 \mathrm{~ms} / 2$ (TE/TR/Nex); acquisition time $3 \mathrm{~min} 28 \mathrm{~s}$ ]

3. Axial fast spin echo (FSE) DP/T2-weighted sequence [FOV $25 \mathrm{~cm}$, matrix $192 \times 256,20-120 \mathrm{~ms} / 3,523 \mathrm{~ms} / 2 /$ 4 (TE/TR/Nex/ETL); acquisition time $4 \min 28 \mathrm{~s}$ ]

Table 1 Biochemical data of our cohort of classical PKU patients.

\begin{tabular}{|c|c|c|c|c|c|c|c|}
\hline Patient & Sex & Age (years) & Phe $(\mu \mathrm{mol} / \mathrm{L})$ & $\mathrm{Phe}_{\text {year }}(\mu \mathrm{mol} / \mathrm{L})$ & $\operatorname{Tyr}(\mu \mathrm{mol} / \mathrm{L})$ & $\operatorname{Tyr}_{\text {year }}(\mu \mathrm{mol} / \mathrm{L})$ & Phe/Tyr $r_{\text {year }}$ \\
\hline 1 & M & 10 & 238 & 423 & 58 & 61 & 7.29 \\
\hline 2 & $\mathrm{~F}$ & 12 & 406 & 394 & 44 & 48 & 9.93 \\
\hline 3 & M & 16 & 413 & 320 & 49 & 44 & 6.96 \\
\hline 4 & $\mathrm{~F}$ & 18 & 374 & 564 & 81 & 40 & 15.37 \\
\hline 5 & $\mathrm{~F}$ & 18 & 343 & 507 & 32 & 50 & 10.68 \\
\hline 6 & M & 18 & 852 & n.a. & n.a. & n.a. & n.a. \\
\hline 7 & $\mathrm{~F}$ & 18 & 422 & 396 & 32 & 47 & 8.75 \\
\hline 8 & $\mathrm{~F}$ & 19 & 336 & 505 & 49 & 51 & 10.40 \\
\hline 9 & M & 20 & 572 & 653 & 26 & 33 & 19.20 \\
\hline 10 & $\mathrm{~F}$ & 22 & 566 & 1,048 & 51 & 33 & 39.07 \\
\hline 11 & M & 22 & 1,004 & 1,103 & 73 & 72 & 16.92 \\
\hline 12 & $\mathrm{~F}$ & 22 & 369 & 724 & 46 & 40 & 18.38 \\
\hline 13 & M & 23 & 670 & 1,195 & 81 & 69 & 19.05 \\
\hline 14 & M & 24 & 623 & 812 & 41 & 33 & 26.03 \\
\hline 15 & M & 25 & 1,166 & 946 & 56 & 43 & 23.04 \\
\hline 16 & M & 25 & 569 & 629 & 55 & 84 & 7.80 \\
\hline 17 & $\mathrm{M}$ & 26 & 909 & 732 & 29 & 32 & 23.13 \\
\hline 18 & $\mathrm{~F}$ & 27 & 454 & 838 & 66 & 56 & 16.65 \\
\hline 19 & M & 29 & 562 & 929 & 30 & 33 & 28.46 \\
\hline 20 & $\mathrm{M}$ & 30 & 900 & 932 & 57 & 50 & 18.86 \\
\hline
\end{tabular}

Phe phenylalanine plasma concentration shortly before MR examination, Phe $e_{\text {year }}$ mean phenylalanine plasma levels collected in the year before MRI, Tyr tyrosine plasma concentration shortly before MR examination, Tyr $r_{\text {year }}$ mean tyrosine plasma concentration during the year before MR examination, $P$ he/Tyr $r_{\text {year }}$ mean phenylalanine-tyrosine concentration ratio in the year preceding MR examination, n.a. not available 
4. Axial FSE FLAIR [FOV 25; matrix $192 \times 256,80 \mathrm{~ms} /$ $6,000 \mathrm{~ms} / 2,100 \mathrm{~ms} / 2 / 8$ (TE/TR/TI/Nex/ETL); acquisition time $4 \mathrm{~min}$ ]

5. Axial SE echo-planar diffusion-weighted sequence (DWI) with $b$ values of 0 and $1,000 \mathrm{~s} / \mathrm{mm}^{2}$ applied in three orthogonal directions [FOV $24 \mathrm{~cm}$, matrix 128× $128,113 \mathrm{~ms} / 6,597 \mathrm{~ms} / 1$ (TE/TR/Nex); acquisition time $39 \mathrm{~s}$ ]; trace images and ADC maps were automatically calculated.

All axial images had the same position, thickness $(5.5 \mathrm{~mm})$ and gap $(1 \mathrm{~mm})$. ADC trace values were measured in the parietal white matter within manually positioned regions of interest.

In 1993, Thompson et al. [6] suggested a dedicated scale to evaluate WMAs on T2-weighted images in PKU patients. According to this scale, WMA were graded semiquantitatively in six degrees based on the percentage of parieto-occipital periventricular WM involvement, on the dimension of isolated WM lesions, and on the presence of septum pellucidum and corpus callosum hyperintensities on T2 images. Scans were graded as follows:

0 Normal or equivocal

1 Minimal periventricular abnormalities with occasional, small and discrete lesions $(<5 \mathrm{~mm})$

2 Up to $30 \%$ parieto-occipital involvement with occasional and discrete lesions $(<5 \mathrm{~mm})$

$330-50 \%$ parieto-occipital involvement with some small-to-medium-sized discrete lesions $(5-10 \mathrm{~mm})$ and septum pellucidum involvement

$4 \quad 50-75 \%$ parieto-occipital involvement with some medium-sized discrete lesions and septum pellucidum and corpus callosum involvement

$5 \quad>75 \%$ parieto-occipital involvement with medium-sized discrete lesions and septum pellucidum and corpus callosum involvement

To obtain more detailed information about supratentorial white matter, particularly in frontal and temporal lobes, we applied another semiquantitative scale which took into account both the extension and the intensity of signal abnormalities in different regions of the brain, that is, the frontal, temporal, occipital, and parietal lobes; the corpus callosum; and the fornix. In each lobe, WMAs ranged from 0 (normal) to 4 according to the percentage of WM involvement:

1. Periventricular abnormalities $<30 \%$

2. Periventricular abnormalities $30-50 \%$

3. Periventricular abnormalities $50-75 \%$

4. Periventricular abnormalities $>75 \%$

The fornix was graded 0 (normal) or 1 (involved) while the corpus callosum was scored as follows: 0 (normal), 1 (either splenium or genu involvement), and 2 (both splenium and genu involvement). Moreover, T2 and DWI WMAs intensity was graded 1 or 2 in each area and a final regional score was obtained by multiplying the intensity and the extension scores.

In the end, all the regional scores of each patient were summed up in order to have a global score for both $\mathrm{T} 2$ and DWI scans (Fig. 1). The neuroradiologists were blinded about the clinical and biochemical status of the patients.

Statistical analysis

Semiquantitative scores obtained by Thompson and modified Thompson scale on T2 images and DWI were analyzed by Wilcoxon and Spearman tests. By using the Spearman test, MRI findings were correlated to Phe and Tyr plasma concentrations measured before MRI examination and to mean plasma phenylalanine ( Phe $_{\text {year }}$ ) levels in the year before MR imaging was performed. The linear correlations between normally distributed variables were tested using the multiple linear regression model. Normality of variables was analyzed using the test $W$ of Shapiro-Wilk.

\section{Results}

The mean Thompson score of cPKU patients was $2.65 \pm 1.4$ and $3.50 \pm 1.7$ on T2 images and DWI, respectively. DWI scores were significantly higher than $\mathrm{T} 2$ ones $(p=0.002$, Wilcoxon test). The mean Thompson score of controls on T2 images was 0.19 with some WMAs probably due to terminal myelination areas, enlarged perivascular VirchowRobin spaces, or to nonspecific gliotic areas. All controls were scored 0 on DWI images.

The mean modified Thompson score of cPKU patients was $15.85 \pm 9.8$ and $23.65 \pm 12.8$ on T2 images and DWI, respectively. DWI scores were significantly higher than T2 ones ( $p=$ 0.0003 , Wilcoxon test). The mean modified Thompson score of controls was $1.7 \pm 2.5$ on T2 images and 0 on DWI images. In each examined region, except for the occipital lobe, DWI scores of cPKU patients were significantly higher than T2 ones $(p<0.01$, Wilcoxon test; Table 2$)$.

A strong linear correlation was found between Thompson and modified Thompson scale applied on T2 images and on DWI $(p<0.0001$ and $p<0.00001$, respectively, Spearman test). According to the modified Thompson scale on T2 images, parietal vs temporal scores tended to show a more severe involvement ( $p=0.098$, Wilcoxon test), while there was no statistical difference between occipital vs parietal or frontal vs temporal scores $(p=0.221$ and $p=0.443$, respectively, Wilcoxon test).

The same scale applied on DWI disclosed an almost significant difference between parietal and occipital scores $(p=0.069$, Wilcoxon test) and a statistical difference 

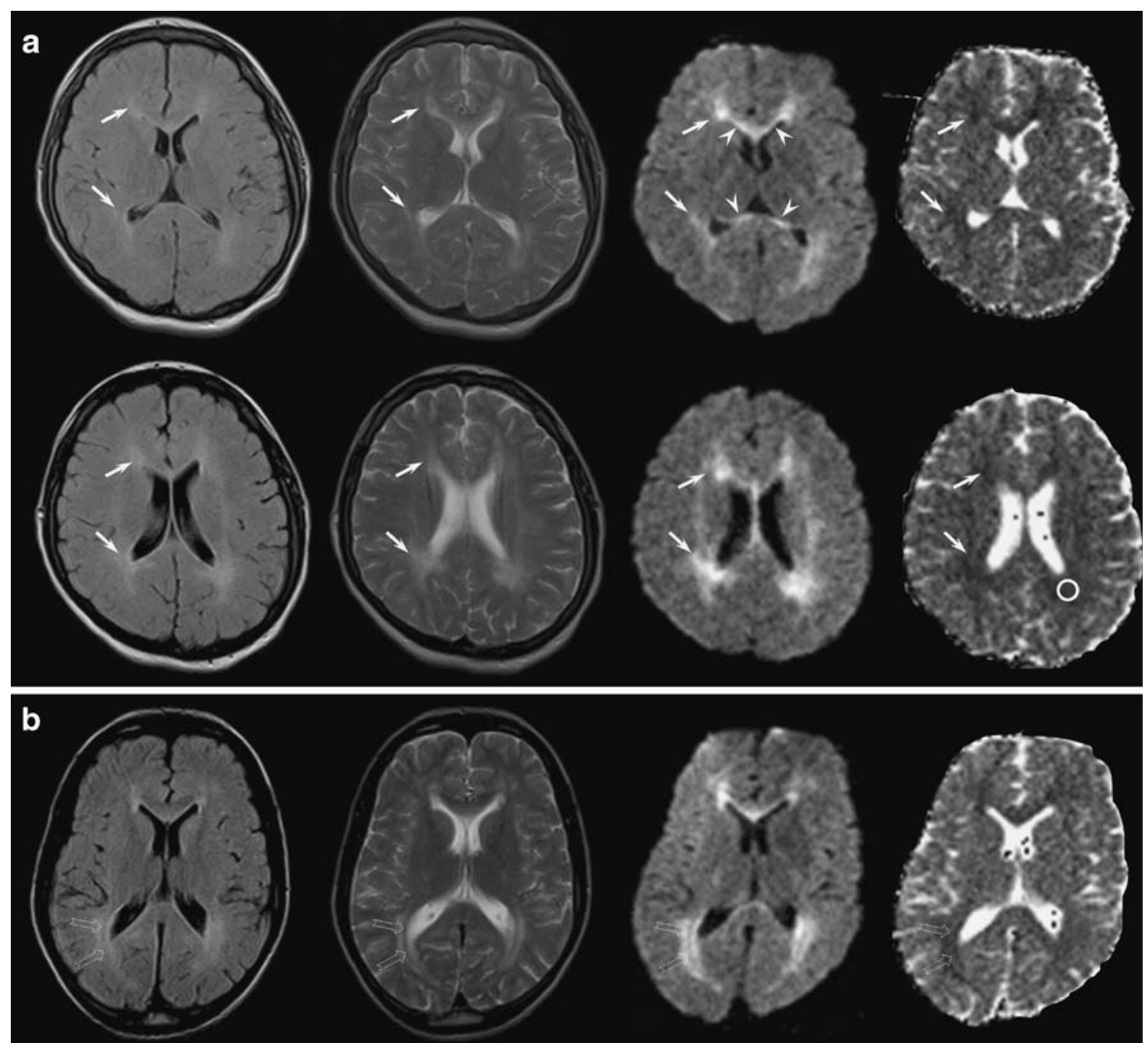

Fig. 1 a, b Two different cPKU patients; each row is composed by axial FLAIR, T2, DWI, and ADC maps at the same level and show typical cPKU high-signal-intensity abnormalities in the parietooccipital and frontal periventricular white matter (white arrows) while apparent diffusion coefficient values in the affected areas are decreased. Splenium and genu of corpus callosum involvement (white

between occipital and temporal scores $(p=0.038$, Wilcoxon test); no difference was found between frontal and temporal scores $(p=0.789$, Wilcoxon test). Therefore, the modified Thompson scale applied on DWI showed a clear tendency arrowheads) is better depicted in DWI than in T2/FLAIR, since partial volume average artifacts may conceal the WMAs; note the characteristic sparing of the optical radiation (open arrows). Measurement within the circular region of interest placed in the left periventricular occipital white matter shows ADC values of $566 \pm 75$ vs $750 \pm 65 \mathrm{~mm}^{2} / \mathrm{s}$ of normal white matter

of the progressive involvement of the frontotemporal, occipital, and parietal regions. In cPKU patients, parietal white matter presented significantly reduced ADC trace values within manually positioned regions of interest
Table 2 Mean of modified Thompson scores in each region examined on $\mathrm{T} 2$ and DWI

All $p$ values in this table were calculated by Wilcoxon test

$* p=0.098$ parietal vs temporal T2 scores; ** $p=0.069$ parietal vs occipital DWI scores; $* * * p=$ 0.038 occipital vs temporal DWI scores

\begin{tabular}{lccccl}
\hline Region & \# of patients & Mean T2 score & Mean DWI score & Score range & $p$ value \\
\hline Parietal & 20 & $4.40 \pm 2.70$ & $6.30 \pm 2.85$ & $0-8$ & $<0.01$ \\
Occipital & 20 & $5.05 \pm 3.07$ & $5.45 \pm 3.17^{* *}$ & $0-8$ & n.s. \\
Temporal & 20 & $3.10 \pm 2.90^{*}$ & $4.35 \pm 3.30^{* * *}$ & $0-8$ & $<0.01$ \\
Frontal & 20 & $2.60 \pm 2.62$ & $4.15 \pm 2.94$ & $0-8$ & $<0.01$ \\
Fornix & 20 & $0.35 \pm 0.67$ & $2.30 \pm 1.45$ & $0-2$ & $<0.01$ \\
Corpus Callosum & 20 & $0.35 \pm 0.59$ & $1.10 \pm 0.85$ & $0-4$ & $<0.01$ \\
Total & 20 & $15.85 \pm 9.8$ & $23.65 \pm 12.8$ & $0-38$ & $<0.01$ \\
\hline
\end{tabular}


$\left(667 \pm 79\right.$ vs $846 \pm 63 \mathrm{~mm}^{2} / \mathrm{s}$ of age matched controls, $p<$ 0.001 , Student's $t$ test).

No WMAs were detected in subjects with Phe levels determined shortly before MR examination lower than $400 \mu \mathrm{mol} / \mathrm{L}$ or with mean $\mathrm{Phe}_{\text {year }}$ values lower than $460 \mu \mathrm{mol} / \mathrm{L}$.

Our patients were subdivided into two groups according to the recommended $\mathrm{Phe}_{\text {year }}$ level (i.e., below or above $700 \mu \mathrm{mol} / \mathrm{L}$ ) and their TS and mTS were compared. The T2 Thompson score did not reach a significant difference between the two groups $(p=0.06)$, while DWI TS and both T2 and DWI mTS were significantly higher in cPKU patients with Phe $_{\text {year }}$ levels above $700 \mu \mathrm{mol} / \mathrm{L}(p=0.001)$.

Correlations between $\mathrm{Phe}, \mathrm{Phe}_{\mathrm{year}}$, and $\mathrm{Phe} / \mathrm{Tyr}_{\mathrm{year}}$ concentrations and semiquantitative scores are shown in Table 3. In particular, no correlation was found between Phe levels collected shortly before MRI examination and T2 Thompson score; on the other hand, the DWI Thompson score showed a strong correlation with Phe concentrations. However, an even stronger correlation was found between DWI-modified Thompson scores and Phe levels.

A linear correlation was found between age and DWI mTS (rho=0.52, $p<0.02$ ). Actually, in our cohort of earlytreated cPKU patients, only subjects older than 14 years showed typical WMAs. Nevertheless, a strong correlation

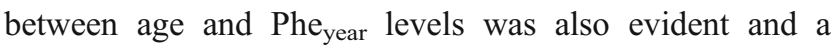
further statistical analysis was performed. The test $W$ of Shapiro-Wilk showed that age, DWI mTS, and Phe $\mathrm{Pear}_{\text {, }}$ levels were normally distributed. So, a multiple regression model was used with DWI mTS as dependent variable and age and $\mathrm{Phe}_{\text {year }}$ level as independent variables. This analysis showed that only $\mathrm{Phe}_{\text {year }}$ was significantly correlated whit DWI mTS (partial correlation: $r=0.68, p<0.002$ ) while age showed only a correlation trend with $\operatorname{mTS}(p=$ $0.1)$. No correlation was found between Tyr plasma levels and $\mathrm{T} 2$ and DWI findings.

\section{Discussion}

In this study, we compared T2 and DWI white matter signal abnormalities in a homogeneous cohort of patients affected with the classical form of PKU, treated from birth with an appropriate low-Phe diet to define the more suitable, specific, and sensitive MR protocol.

Our findings consistently show that DWI is more sensitive than T2 images for the evaluation of white matter involvement in cPKU patients since DWI scores were almost always significantly higher than T2 scores.

WMAs and plasma levels of phenylalanine and tyrosine

The supratentorial brain white matter is known to be primarily involved in phenylketonuria as has already been extensively reported in children, adolescents, and adults with PKU [3-5, 14], even in patients with an early strict dietary control. Interestingly, in a recent paper, Koch and coworkers reported delay of myelination (all lobes), at autopsy, in a 4-month-old boy born to a woman with maternal PKU and high Phe levels during pregnancy [15], thus underlying the link between high blood levels of Phe and white matter involvement.

In our study, phenylalanine plasma levels directly correlated to the presence of WMAs on T2 and on DWI.

Recommendations for treatment are based on age-related blood Phe levels which are, however, different according to national standards. In our clinical practice, due to the lack of national recommendations, our treatment strategy was lifted from different authors, and we use, as safety levels, Phe below $360 \mu \mathrm{mol} / \mathrm{L}$ for children under the age of 6 , below $480 \mu \mathrm{mol} / \mathrm{L}$ for patients under the age of 12 and below $700 \mu \mathrm{mol} / \mathrm{L}$ for adolescents and adults. These levels are considered to be safe in order to prevent clinical symptoms in cPKU patients but do not seem to avoid the
Table 3 Relationship between Phe levels and both Thompson and modified Thompson scores calculated on T2 and DWI.

\# $c P K U$ patients number of patients affected by the classical form of phenylketonuria, Phe phenylalanine plasma concentrations before MR examination, Phe year mean phenylalanine plasma levels collected in the year before MRI, Phe/Tyr $r_{\text {year }}$ mean phenylalanine-tyrosine concentration ratio in the year preceding MR examination

\begin{tabular}{|c|c|c|c|}
\hline & \# cPKU patients & Spearman & $p$ \\
\hline Phe vs modified Thompson T2 score & 19 & 0.71 & $<0.001$ \\
\hline Phe vs modified Thompson DWI score & 19 & 0.71 & $<0.001$ \\
\hline Phe vs Thompson T2 score & 19 & 0.38 & 0.103 \\
\hline Phe vs Thompson DWI score & 19 & 0.67 & 0.001 \\
\hline Phe $_{\text {year }}$ vs modified Thompson T2 score & 19 & 0.76 & $<0.001$ \\
\hline Phe $_{\text {year }}$ vs modified Thompson DWI score & 19 & 0.81 & $<0.001$ \\
\hline Phe $_{\text {year }}$ vs Thompson T2 score & 19 & 0.55 & 0.013 \\
\hline Phe $_{\text {year }}$ vs Thompson DWI score & 19 & 0.78 & $<0.001$ \\
\hline Phe/Tyr ${ }_{\text {year }}$ vs modified Thompson T2 score & 19 & 0.81 & $<0.001$ \\
\hline Phe/Tyr $r_{\text {year }}$ vs modified Thompson DWI score & 19 & 0.82 & $<0.001$ \\
\hline Phe/Tyr ${ }_{\text {year }}$ vs Thompson T2 score & 19 & 0.57 & 0.010 \\
\hline Phe/Tyr ${ }_{\text {year }}$ vs Thompson DWI score & 19 & 0.84 & $<0.001$ \\
\hline
\end{tabular}


appearance of WMAs. In our cohort, not only all patients with Phe $_{\text {year }}$ above the recommended plasma concentrations presented WMAs but also six out of nine patients, despite the lower Phe levels, showed WMAs. No WMAs were seen in subjects with Phe levels measured shortly before MR examination lower than $400 \mu \mathrm{mol} / \mathrm{L}$ or in those with mean Phe $_{\text {year }}$ lower than $460 \mu \mathrm{mol} / \mathrm{L}$. A positive correlation between the presence of WMAs and plasma Phe levels has already been reported by other authors [2-5, 8, 13] but a clear Phe cutoff value has not been identified yet. Kono et al. [11] reported the absence of WMAs and decreased ADCs in the deep white matter in PKU patients with plasma phenylalanine levels below $8.5 \mathrm{mg} / \mathrm{dL}(514.3 \mu \mathrm{mol} / \mathrm{L})$. $\mathrm{n}$ our cohort, however, four out of nine patients with plasma Phe levels below that value showed the typical PKU MRI changes. These findings are apparently unexpected considering the heterogeneity of Kono's sample, where only 14 of the 21 patients were diagnosed as having PKU from birth, while seven were born before the introduction of neonatal mass screening and therefore were treated later in life. Our cohort of cPKU patients was strictly homogeneous, since PKU was diagnosed at birth and patients were always under strict dietary control till adolescence, thus minimizing the variability of blood Phe concentrations. The increased sensitivity of MRI in our patients, even with Phe levels lower than $8.5 \mathrm{mg} / \mathrm{dL}$, may be due to the use of a dedicated score which allows for an easier detection of WMAs on DWI rather than on ADC maps. As a matter of fact, even if ADC values remain an important MR parameter in the evaluation of normal-appearing brain tissue of PKU patients, as recently demonstrated by Ding et al. [16], a slight reduction of ADC values may be hardly recognized. Difficulties in defining a one-size-fits-all plasma Phe cutoff may also be related to the interindividual variability in the affinity of the transporter of phenylalanine, so that different brain Phe levels can be found in patients with similar blood Phe concentrations [3]. That is why we believe that the search for a precise cutoff for plasma Phe in relationship to ADC values or T2 images might be disappointing, while DWI could represent a more sensitive indicator of WM involvement even in asymptomatic patients.

In this study, Tyr and $\mathrm{Tyr}_{\text {year }}$ concentrations do not correlate with the amount of WMAs. Therefore, even though a correlation between low levels of plasma tyrosine and reduced cerebrospinal fluid (CSF) biogenic amine neurotransmitters (i.e., dopamine and serotonin) have been previously reported [17], WMAs are not likely to depend on plasma tyrosine deficiency.

\section{WMAs and age}

In our cohort of early-treated cPKU patients, no patients younger than 14 presented brain lesions even though other authors reported these abnormalities in two children of 7 and 10 years old, respectively [10].

Interestingly, all our cPKU patients with an age range between 10 and 30 years had either normal brain MRI findings or presented a restricted water diffusion (i.e., reduced $\mathrm{ADC}$ values) within the WMAs; in particular, none of them showed increased ADC values within WMAs. This homogeneous brain MR picture, resulting from a relatively large cohort of adolescent/adult cPKU patients, seems to be in contrast with Sener's study [10] which describes two different PKU lesion patterns in both 7- and 10-year-old PKU patients (one with restricted and the other with increased diffusion). He suggested that these patterns reflected two different histopathological changes or stages of the disease. Intriguingly, the 10-year-old patient with increased ADC values described by Sener [10] presented an atypical lesion pattern in terms of site and extension, which in normal young subjects is usually referred to as nonspecific gliosis or terminal areas of myelination. It is interesting to note that in our control group, we also found a subject with an asymptomatic frontal gliotic area. Therefore, although gliosis is a widely described histopathological finding in the periventricular white matter of untreated PKU patients, it is questionable if this can be related to the disease itself or whether it can be considered as an aspecific MRI finding in early-treated PKU patients.

WMAs and semiquantitative analysis on T2-weighted MRI and diffusion-weighted imaging

Although no correlation has yet been found between WM involvement and neurological findings, our knowledge of the natural history of early-treated cPKU patients is relatively limited [7], since neonatal screening was introduced in our country only in the early 1970s. For this reason, we still need to follow up this new cohort of earlytreated patients as we simply do not know what could be their clinical evolution. Furthermore, cognitive and neurophysiologic tests, such as event-related potentials, have revealed that adults with PKU present subtle impairments even if treated from birth [18, 19], but according to other reports, evoked potentials showed a limited utility in the follow-up of the disease [20]. Therefore, there is a need for other technical tools for monitoring the progression of the disease and we believe that a semiquantitative MRI analysis could be a reproducible and useful tool to follow up earlytreated cPKU patients.

The scale dedicated to T2-WMA evaluation available at the moment was proposed by Thompson in 1993 [6]. He suggested a semiquantitative score which showed a significant correlation between plasma Phe levels and the degree of WM involvement in the posterior regions of the cerebral hemispheres (see "Materials and methods" section 

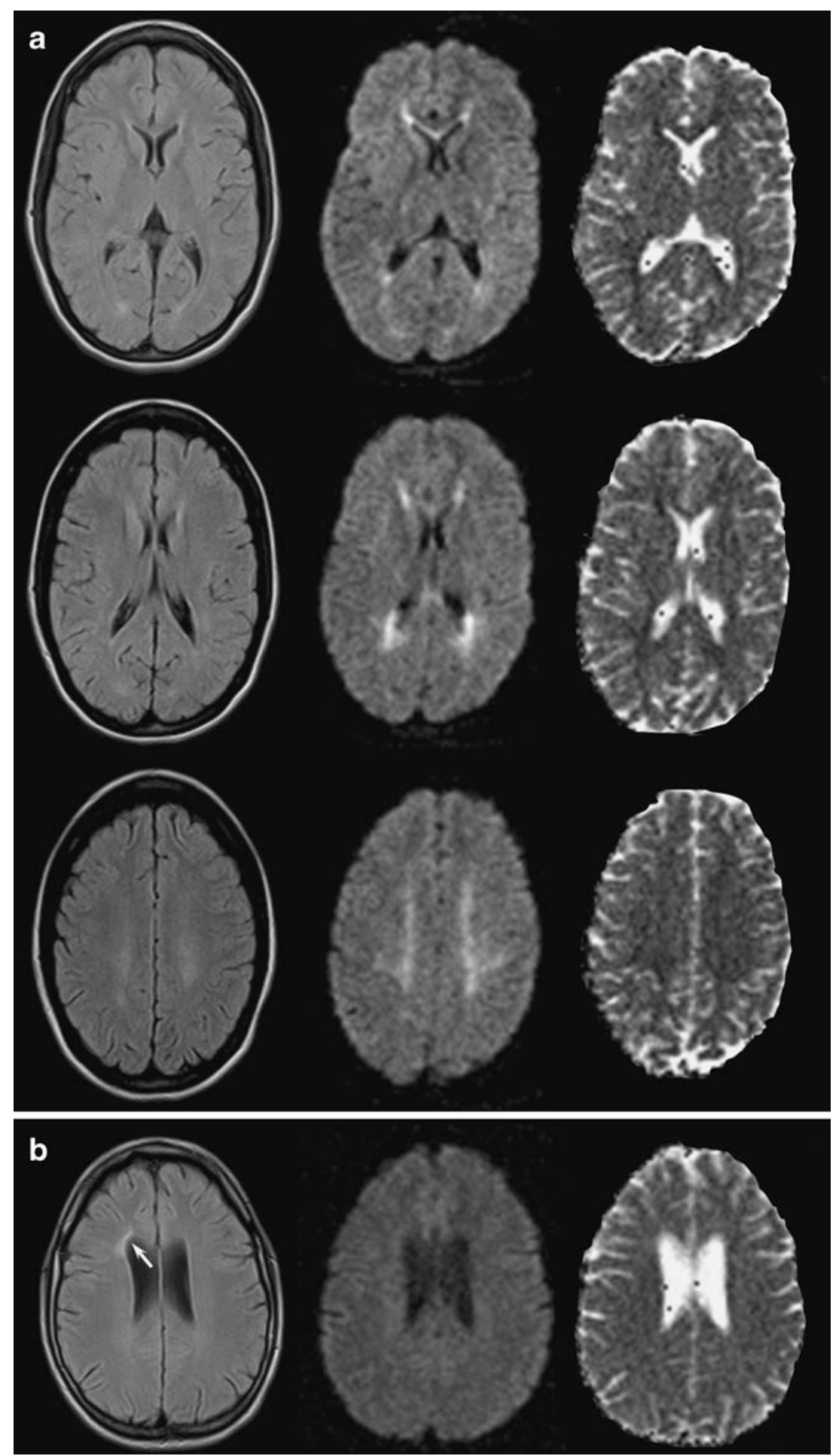

Fig. 2 Axial images at the level of the basal ganglia, of the corona radiata, and above the lateral ventricles; DWI, FLAIR, and ADC maps images share the same level and thickness. a cPKU patient: Typical WMAs are more evident on DWI rather than T2/FLAIR sequences. Affected areas present decreased ADC values but signal abnormalities are less recognizable on ADC maps due to relatively low signal-to- noise ratio of these sequences. b Control subject with an occasional finding of right frontal periventricular gliosis (white arrows) of unknown origin; FLAIR discloses a hyperintense region (white arrows) which is not detected by DWI, thus showing the higher specificity of DWI for cPKU-related WMAs; ADC values were increased within this area $\left(935 \pm 70 \mathrm{~mm}^{2} / \mathrm{s}\right)$ 
for detailed description). In the present study, we applied this score also to DWI, showing an even higher correlation to plasma Phe concentration as well as a higher specificity of DWI compared to T2 images.

In fact, increased signal intensity in cerebral WM on $\mathrm{T} 2$ images can be caused by several factors, including delayed myelination and enlargement of Virchow-Robin perivascular spaces or nonspecific focal gliosis. DWI had the ability to distinguish typical PKU WM changes, characterized by restricted diffusivity (i.e., increased DWI signal with reduced ADC values), from the other aforementioned conditions (isohypointense in DWI with increased ADC values). Indeed, DWI scans of controls evaluated using the Thompson scale were always scored 0 with no false positives, whereas on T2 images, some control subjects presented nonspecific periventricular WMAs, thus underlying the higher specificity of DWI compared to T2 images. This aspect can be very important considering the increasing age of early-treated cPKU patients and the possibility that WM hyperintensities during aging could depend on other kinds of damage (for example, chronic ischemia or age-related WM changes; Fig. 2). In order to further improve reliability and sensitivity in detecting WMA changes, we developed a dedicated MRI scale which included the criteria suggested by Thompson, graded the intensity of WMAs, and considered the involvement of the frontal and temporal regions.

The modified Thompson scale applied to DWI allowed us to detect the progressive involvement of the temporal and frontal, occipital, and parietal periventricular white matter regions, previously suggested in other papers $[3,5]$. Furthermore, the DWI sequence, in comparison to T2 sequence, showed more clearly abnormalities both in the fornix and in the corpus callosum, which frequently may present partial averaging artifacts in T2 images. In these regions, T2 hyperintense lesions may be hardly recognized due to their proximity to the T2 highly hyperintense CSFfilled supratentorial ventricles. This problem can be overcome with DWI since cPKU hyperintense WMAs are easily distinguishable from the contiguous hypointense ventricles (Fig. 1).

Moreover, MR DWI and T2 scores calculated according to this new scale showed a very high correlation with both recent and chronic plasma Phe concentrations. Previous studies [1, 3, 9] quantifying WMAs on MRI suggested that, rather than the quality of a long-term control, it was the exposure to high Phe concentrations shortly before the neuroradiological examination that significantly determined the typical lesions. Our data do not support this observation, since the correlation seems to be a little stricter with chronic exposure to Phe high levels. This suggests that the quality of a long-term phenylalanine control is the primary determinant of MRI changes, and this underlines the highly possible causative role of phenylalanine or its metabolites in the pathogenesis of WMAs.

\section{Conclusions}

Our data provide further evidence of a lifelong susceptibility of the nervous system in subjects with classical PKU, which correlates with the severity of prolonged hyperphenylalaninemia. Because of the increase in Phe intake, due to the difficulties in following a strict diet, and consequent high blood Phe levels during adulthood, we could expect a late brain involvement during the patients' life. Semiquantitative MRI scores, particularly by means of DWI which combines the effect of $\mathrm{T} 2$ prolongation (T2 shine-through) and ADC reduction, might be a useful, reproducible, and reliable tool in the follow-up of classical PKU patients who show brain MRI white matter abnormalities. Therefore, on the basis of this study, we believe that brain MRI, especially DWI, should be included in the long-term follow-up of classical PKU patients.

Acknowledgment The authors thank Prof. Reuben Matalon for review of the paper and helpful comments.

Conflict of interest statement We declare that we have no conflict of interest.

\section{References}

1. Dezortova M, Hajek M, Tintera J et al (2001) MR in phenylketonuria-related brain lesions. Acta Radiol 42:459-466

2. Koch R, Moats R, Guttler F et al (2000) Blood-brain phenylalanine relationships in persons with phenylketonuria. Pediatrics 106:1093-1096

3. Van der Knaap MS, Valk J (2005) Phenylketonuria. In: van der Knaap MS, Valk J (eds) Magnetic resonance of myelination and myelin disorders. Springer, Berlin, pp 284-293

4. Leuzzi V, Trasimeni G, Gualdi GF et al (1995) Biochemical, clinical and neuroradiological MRI correlations in late-detected PKU patients. J Inherit Metab Dis 18:624-634

5. Leuzzi V, Tosetti M, Montanaro D (2007) The pathogenesis of the white matter abnormalities in phenylketonuria. A multimodal 3.0 tesla MRI and magnetic resonance spectroscopy (1 H MRS) study. J Inherit Metab Dis 30:209-216

6. Thompson AJ, Tilloston S, Smith I et al (1993) Brain MRI changes in phenylketonuria. Associations with dietary status. Brain 116:811-821

7. Vermathen P, Robert-Tissot L, Pietz J et al (2007) Characterization of white matter alterations in phenylketonuria by magnetic resonance relaxometry and diffusion tensor imaging. Magn Reson Med 58:1145-1156

8. Moller HE, Weglage J, Bick U et al (2003) Brain imaging and proton magnetic resonance spectroscopy in patients with phenylketonuria. Pediatrics 112:1580-1583

9. Phillips MD, Mc Graw P, Lowe MJ et al (2001) Diffusionweighted imaging of white matter abnormalities in patients with phenylketonuria. Am J Neuroradiol 22:1583-1586 
10. Sener RN (2003) Diffusion MRI findings in phenylketonuria. Eur Radiol 13:L226-L229

11. Kono K, Okano Y, Nakayama K et al (2005) Diffusion-weighted MR imaging in patients with phenylketonuria: relationship between serum phenylalanine levels and ADC values in cerebral white matter. Radiology 236:630-636

12. Sirrs SM, Laule C, Madler B (2007) Normal-appearing white matter in patients with phenylketonuria: water content, myelin eater fraction, and metabolite concentrations. Radiology 242:236-243

13. Bauman ML, Kemper TL (1982) Morphologic and histoanatomic observations of the brain in untreated human phenylketonuria. Acta Neuropathol 58:55-63

14. Huttenlocher (2000) The neuropathology of phenylketonuria: human and animal studies. Eur J Pediatr 159:102-106

15. Koch R, Verma S, Gilles FH (2008) Neuropathology of a 4-month-old infant born to a woman with phenylketonuria. Dev Med Child Neurol 50:230-233
16. Ding X-Q, Fiehler J, Kohlschütter B et al (2008) MRI abnormalities in normal-appearing brain tissue of treated adult PKU patients. J Magn Reson Imaging 27:998-1004

17. Burlina AB, Bonafè L, Ferrrari V et al (2000) Measurement of neurotransmitter metabolites in the cerebrospinal fluid of phenylketonuric patients under dietary treatment. J Inherit Metab Dis 23:313-316

18. Anderson PJ, Wood SJ, Francis DE et al (2007) Are neuropsychological impairments in children with early-treated phenylketonuria (PKU) related to white matter abnormalities or elevated phenylalanine levels? Dev Neuropsychol 32:645-668

19. Moyle JJ, Fox AM, Bynevelt M et al (2006) Event-related potentials elicited during a visual Go-Nogo task in adults with phenylketonuria. Clin Neurophysiol 117:2154-2160

20. Leuzzi V, Cardona F, Antonozzi I et al (1994) Visual, auditory, and somatosensorial evoked potentials in early and late treated adolescents with phenylketonuria. J Clin Neurophysiol 11:602-606 